\title{
ARTICLE
}

\section{Neurobiology of loneliness: a systematic review}

\author{
Jeffrey A. Lam ${ }^{1}$, Emily R. Murray ${ }^{2,3}$, Kasey E. Yu ${ }^{3}$, Marina Ramsey (D) ${ }^{3}$, Tanya T. Nguyen ${ }^{3,4,5}$, Jyoti Mishra ${ }^{4}$, Brian Martis ${ }^{4,5}$, \\ Michael L. Thomas ${ }^{6}$ and Ellen E. Lee Th, $^{3,4}$
}

This is a U.S. government work and not under copyright protection in the U.S.; foreign copyright protection may apply 2021

Loneliness is associated with increased morbidity and mortality. Deeper understanding of neurobiological mechanisms underlying loneliness is needed to identify potential intervention targets. We did not find any systematic review of neurobiology of loneliness. Using MEDLINE and PsycINFO online databases, we conducted a search for peer-reviewed publications examining loneliness and neurobiology. We identified 41 studies ( $n=16,771$ participants) that had employed various methods including computer tomography (CT), structural magnetic resonance imaging (MRI), functional MRI (fMRI), electroencephalography (EEG), diffusion tensor imaging (DTI), single-photon emission computed tomography (SPECT), positron emission tomography (PET), and postmortem brain tissue RNA analysis or pathological analysis. Our synthesis of the published findings shows abnormal structure (gray matter volume or white matter integrity) and/or activity (response to pleasant versus stressful images in social versus nonsocial contexts) in the prefrontal cortex (especially medial and dorsolateral), insula (particularly anterior), amygdala, hippocampus, and posterior superior temporal cortex. The findings related to ventral striatum and cerebellum were mixed. fMRI studies reported links between loneliness and differential activation of attentional networks, visual networks, and default mode network. Loneliness was also related to biological markers associated with Alzheimer's disease (e.g., amyloid and tau burden). Although the published investigations have limitations, this review suggests relationships of loneliness with altered structure and function in specific brain regions and networks. We found a notable overlap in the regions involved in loneliness and compassion, the two personality traits that are inversely correlated in previous studies. We have offered recommendations for future research studies of neurobiology of loneliness.

Neuropsychopharmacology (2021) 46:1873-1887; https://doi.org/10.1038/s41386-021-01058-7

\section{INTRODUCTION}

Loneliness is a critical determinant of well-being and also a grand challenge to society [1, 2]. Defined as distress due to perceived discrepancy between desired and existing social relationships, loneliness is associated with higher rates of cardiovascular disorders [3], dementia [4], anxiety, depression, suicidal ideation $[5,6]$, and $30 \%$ greater mortality [7-9]. Loneliness is distinct from objective social isolation or the lack of social relationships/contacts. The National Academies of Science, Engineering, and Medicine recently published a report on social isolation and loneliness among older adults, calling for more research of neurobiology and interventions [2]. During the COVID-19 pandemic, loneliness, which has been linked to physical distancing measures, is a growing concern for all age groups across the world.

Humans are a social species and have ingrained neural, hormonal, and genetic mechanisms to help navigate social connections. Absence of quality relationships threatens health and reproduction [10]. Cacioppo et al. posited loneliness evolved to improve survivability when socially isolated, through hypervigilance and increasing motivation to connect with others [10]. Animal models of social isolation have demonstrated alterations in neurotransmitters, receptor sensitivities, and levels of certain biomarkers $[10,11]$. Few studies have examined the impact of social isolation on specific brain regions [11-13]. Furthermore, the subjective nature of loneliness as well as inter-species differences in social functioning and brain structure limit the applicability of the animal studies to the uniquely human state of loneliness [11].

Our recent investigations have found a strong and consistent inverse correlation between the personality traits of loneliness and wisdom, especially the empathy/compassion component of wisdom [14-17]. In contrast to loneliness, wisdom is associated with better mental and physical health [18-20]. The prefrontal cortex and limbic striatum reportedly play a major role in the neurobiology of empathy/compassion and wisdom [21]. Identifying neurobiological mechanisms underlying loneliness is critical for understanding how loneliness contributes to poor mental and physical health and for conceptualizing potential pharmacological and neurostimulation targets. Therefore, we conducted a systematic review to identify and synthesize published brainbased findings linked to loneliness.

\section{METHODS}

\section{Search strategy}

We conducted a literature search for peer-reviewed publications examining loneliness and neurobiology, outlined in the Preferred Reporting Items

\footnotetext{
${ }^{1}$ Warren Alpert Medical School of Brown University, Providence, RI, USA. ${ }^{2}$ Division of Biological Sciences, University of California San Diego, La Jolla, CA, USA. ${ }^{3}$ Sam and Rose Stein Institute for Research on Aging, University of California San Diego, La Jolla, CA, USA. ${ }^{4}$ Department of Psychiatry, University of California San Diego, La Jolla, CA, USA. ${ }^{5}$ Veterans Affairs San Diego Healthcare System, San Diego, CA, USA. ${ }^{6}$ Department of Psychology, Colorado State University, Fort Collins, CO, USA. ${ }^{\circledR}$ email: eel013@health.ucsd.edu
} 


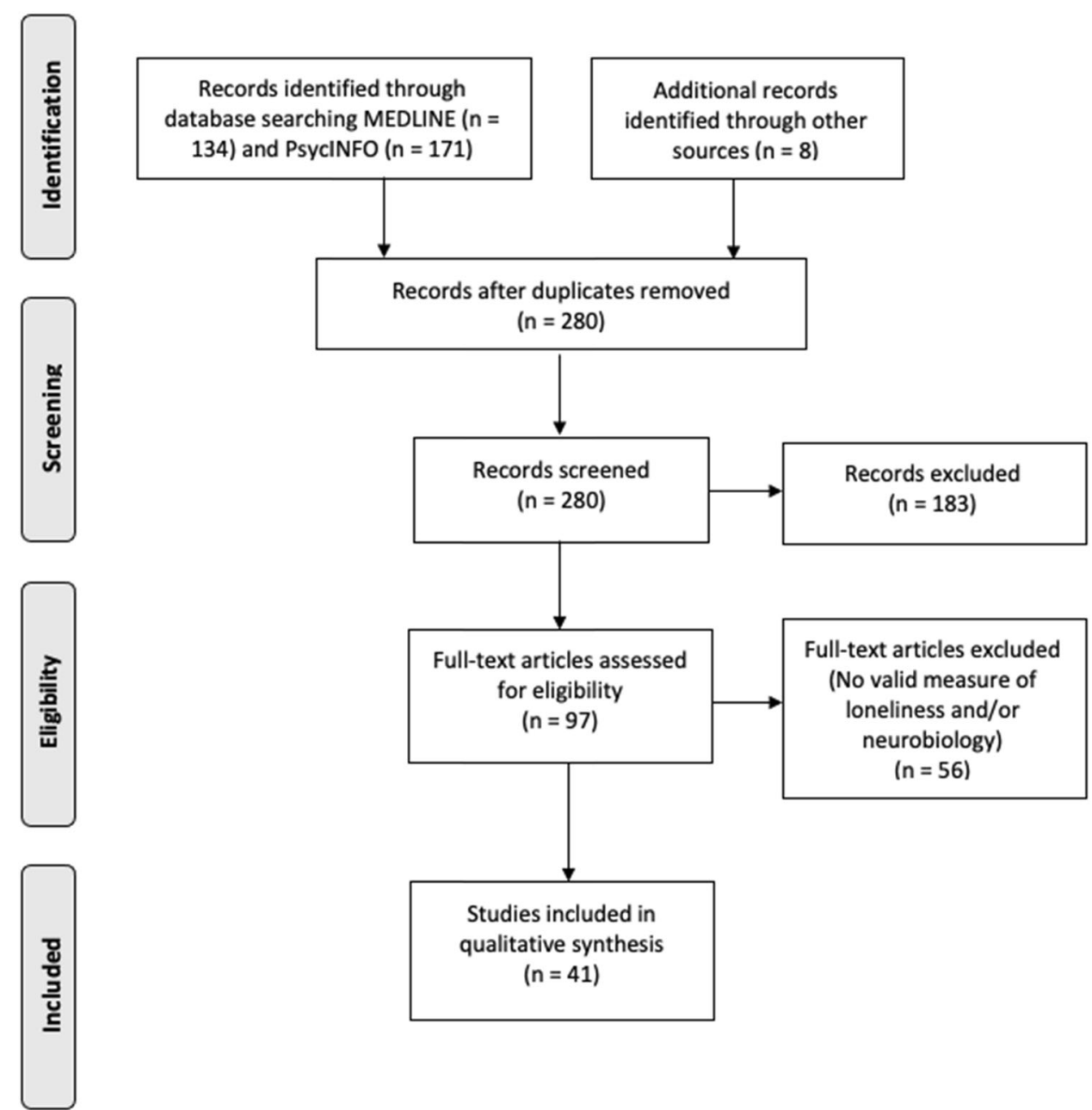

Fig. 1 PRISMA flow diagram for this systematic review that details the database searches, number of abstracts screened, and full-text articles evaluated for this literature review.

for Systematic Reviews and Meta-Analyses (PRISMA) diagram (Fig. 1). We surveyed MEDLINE and PsycINFO online databases on September 24, 2020, with the following inclusion criteria: (1) use of a validated scale for assessing loneliness and a measure of neurobiology, (2) published in English, (3) minimum of 10 human participants, and (4) statistical analysis examining the relationship of loneliness and neurobiology. We excluded animal studies and literature reviews.

We defined validated measures of loneliness as scales or questions that measured feeling lonely, socially isolated, or disconnected. The most commonly used scale was the University of California Los Angeles Loneliness Scale (UCLA-LS [15, 17, 22]), although we also included validated, briefer multiple- or single-item questions [23]. Neurobiology measures included assessments of brain structure or function: computed tomography (CT), magnetic resonance imaging (MRI), functional MRI (fMRI), diffusion tensor imaging (DTI), positron emission tomography (PET), single-photon emission computed tomography (SPECT), or electroencephalography (EEG). We also included brain pathology studies and genetic investigations that extracted genetic materials from brain regions. We did not include studies with only cognitive measures or studies using cortisol or other peripheral biomarkers from blood or other tissues outside the brain. The specific search strategy is outlined in Supplementary Appendix A.

The search yielded 305 articles of interest. After removing duplicates and adding potentially relevant papers from bibliographies of the articles selected, each study title and abstract was screened for eligibility by at least two authors (JAL, ERM, KEY, MR). Articles with any uncertainties were discussed and resolved among all authors. Data from each of the final batch of 41 studies selected (Fig. 1) were extracted by the primary author and checked by at least one other coauthor. Sample sizes ranged from 19 to 10,129 . Most of the studies (61\%) included fewer than 100 individuals, $38 \%$ with 100-942 individuals, and one study with over 10,000 individuals.
To assess the quality of the studies, we used the Joanna Briggs Institute appraisal checklist for cross-sectional studies and cohort studies and Newcastle-Ottawa Scale for case-control studies (Supplementary Table 2).

\section{RESULTS}

\section{Study participant characteristics}

Twenty-four studies focused on younger adults (mean age 18-60; [24-47]), 12 on older adults (mean age >60; [48-59]), two on adolescents $[60,61]$, and two across the lifespan $[62,63]$. Most reports included healthy individuals while seven focused on clinical populations: four with depression $[47,49,51,57]$, and one each with traumatic brain injury (TBI) [58], schizophrenia [28], and severe hearing impairment [27]. Eighteen studies came from the US, 13 from China, three each from Germany and Taiwan, and two from the UK, and one each from Japan and the Netherlands. Of the 41 studies, $5(12.2 \%)$ had hypothesis-driven analyses (e.g., region of interest focused), 21 (51.2\%) had exploratory analyses (e.g., whole brain analyses), 10 (24.4\%) had both, with 5 studies not fitting into any of the above categories.

Fifteen studies analyzed the relationship between loneliness and brain structures using CT [58] or MRI. Twelve were crosssectional, with eight focusing on gray matter volume, $[26,31,35,36,46,57,59,63]$, and four on white matter features, employing DTI or diffusion MRI $[29,32,38,61]$. The three longitudinal investigations included a randomized controlled trial (RCT) of effects of exercise on gray matter volume [54], a 
prospective cohort study of progression of white matter hyperintensities [53], and a study of TBls localized to different brain areas [58]. These study findings are summarized in Table 1.

Eighteen reports analyzed the relationship between loneliness and brain function or connectivity using $\mathrm{fMRI}$. Ten of these studies were task-based ( $n=10 ;[24,25,28,34,44,45,51,60,62,63])$, and two were resting-state fMRI $(n=8 ;[37,39-43,47,49])$ (Table 2). One report appears in both Tables 1 and 2 [63]

Three investigations used EEG to examine high-density eventrelated potentials (ERPs) during different tasks [30, 33, 64], two analyzed RNA expression of post-mortem brain tissue [52, 55], two employed PET to analyze amyloid and tau proteins $[50,56]$, one longitudinal cohort study examined the association between postmortem brain tissue and Alzheimer's disease [48], and one used SPECT to analyze dopamine release in the brain [27] (Table 3).

The quality of the included cross-sectional studies varied primarily on detailed descriptions of the study sample and setting, identification of confounding factors, and use of appropriate statistical strategies for confounders (Supplementary Table 2). The quality of cohort studies varied primarily on representative case sampling and controlling for confounders. There was only one identified cohort study.

\section{Brain regions}

Supplementary Table 2 lists publications sorted by brain regions studied.

Prefrontal cortex or PFC ( $N=14$ studies). Two articles focused on overall PFC. In one, male veterans with TBI to the right PFC had lower levels of loneliness compared to healthy controls [58]. An RCT examining effects of exercise on loneliness in older adults found a greater reduction in stress and loneliness in participants with a larger baseline PFC volume, although PFC volume did not change over the 6-month intervention [54].

In seven studies of medial PFC (mPFC), loneliness was associated with greater MPFC activation in task-based fMRI during a social exclusion paradigm [24], less similarity between selfrepresentation and other-representation in mPFC activation [44], lower dorsomedial PFC (dmPFC) white matter density [32], lower left dmPFC response when looking at pleasant social images and greater left dmPFC response when examining nonsocial images [25], increased functional connectivity between dmPFC and inferior parietal cortex during a working memory task [45], reduced vmPFC activation when looking at images of themselves [60], and nonsignificantly greater gray matter volumetric deviations of the vmPFC in females compared to males [46].

In five reports on dorsolateral PFC (dIPFC), loneliness was associated with increased gray matter in left dIPFC [31], partially mediated the negative association between gray matter volume in left dIPFC and attitudes toward suicide [35], and was associated with lower gray matter volume in dIPFC, especially in subjects aged 69-82 compared to those 61-69 years old and in individuals with depression compared to nondepressed subjects [59]. A resting-state fMRI study found the dIPFC as a node in the predictive model of loneliness [41]. Another dIPFC RNA study is discussed below [55].

Insula $(N=6)$. Investigators reported an association of loneliness with a lesion in the right insula [58], lower gray matter volume, which was even lower in individuals with depression [59], lower regional white matter density in anterior insula [32], and poorer white matter tract connectivity with the nodes in ventral attentional network [29]. fMRI paradigms showed that among lonely individuals, activation of insula (especially anterior insula) was greater among adults looking at pleasant social (than pleasant nonsocial) images, while ventral striatum activation was greater among non-lonely individuals [25]. Among persons with schizophrenia, insula responsiveness was positively correlated with levels of loneliness, while overall insula activation with faces expressing disgust was decreased [28].

Amygdala $(N=6)$. One investigation reported a positive correlation between left amygdala gray matter volume and social distress score, which was mediated by loneliness [36]. Another found that loneliness was associated with lower gray matter volume in left amygdala, especially in subjects aged 61-70 (compared to ages 70-82) [59]. In an RCT of group exercise to improve loneliness among older adults, participants with larger baseline amygdala volumes experienced greater reductions in loneliness [54]. Kiesow et al. found nonsignificantly greater gray matter volumetric deviations of amygdala between lonely and non-lonely males compared to females. [46]. An fMRI region of interest (ROI) analysis failed to find significant differences in amygdala response to social stimuli in young or old adults [62], while another fMRI study found loneliness was associated with a weaker amygdala to superior frontal gyrus connectivity [51].

Ventral striatum/nucleus accumbens $(N=5)$. Studies of ventral striatum response to images with task-based $\mathrm{FMRI}$ paradigms among lonely individuals (vs. non-lonely individuals) reported different results: reduced response to pleasant social (compared to pleasant nonsocial) images [25]; greater response to images of close others (compared to strangers) [34]; and no significant differences in response to pleasant and non-pleasant social and nonsocial images [62]. One report on association of loneliness with gray matter volume in left striatum among older adults with latelife depression found a positive correlation in single depressive episode individuals and negative association in multiple depressive episode individuals [57]. Another nucleus accumbens RNA study is discussed below [52].

Posterior superior temporal cortex $(N=4)$. We defined this region as including both posterior superior sulcus (temporal-parietal junction or TPJ) and the region immediately below it, superior temporal gyrus. Studies reported an association of loneliness with lower white matter regional density [32], less gray matter volume in left posterior superior sulcus [26], lower structural local efficiency in the bilateral superior temporal gyrus [61], and lower bilateral superior temporal gyrus response when looking at unpleasant social images and greater response when looking at unpleasant nonsocial images [25].

Hippocampus $(N=3)$. Investigators reported an association of loneliness with reduced anterior hippocampus gray matter volume, especially in older adults [59], lower white matter local structural efficiency (i.e., shorter weighted paths between local nodes) [61], and greater hippocampal response during a social exclusion task [24].

Cerebellum $(N=3)$. One study reported that loneliness was associated with lower left cerebellar gray matter volume [59]; however, another report found no main effects of loneliness on cerebellar ROIs [63]. Loneliness was associated with higher connectivity between cerebellum and visual cortex during an fMRI Stroop task using positive words [63], and lower local structural efficiency in the white matter of the posterior cerebellum on structural MRI [61].

\section{Networks}

Visual systems $(N=5)$. Investigators demonstrated that loneliness was associated with increased activation of left primary visual cortex and right secondary visual cortex when presented with unpleasant social (compared to unpleasant nonsocial) images [25], differences in the connection of visual network (fusiform gyrus, calcarine fissure, lingual gyrus, middle occipital gyrus, cuneus, superior occipital gyrus, and inferior occipital gyrus) to 


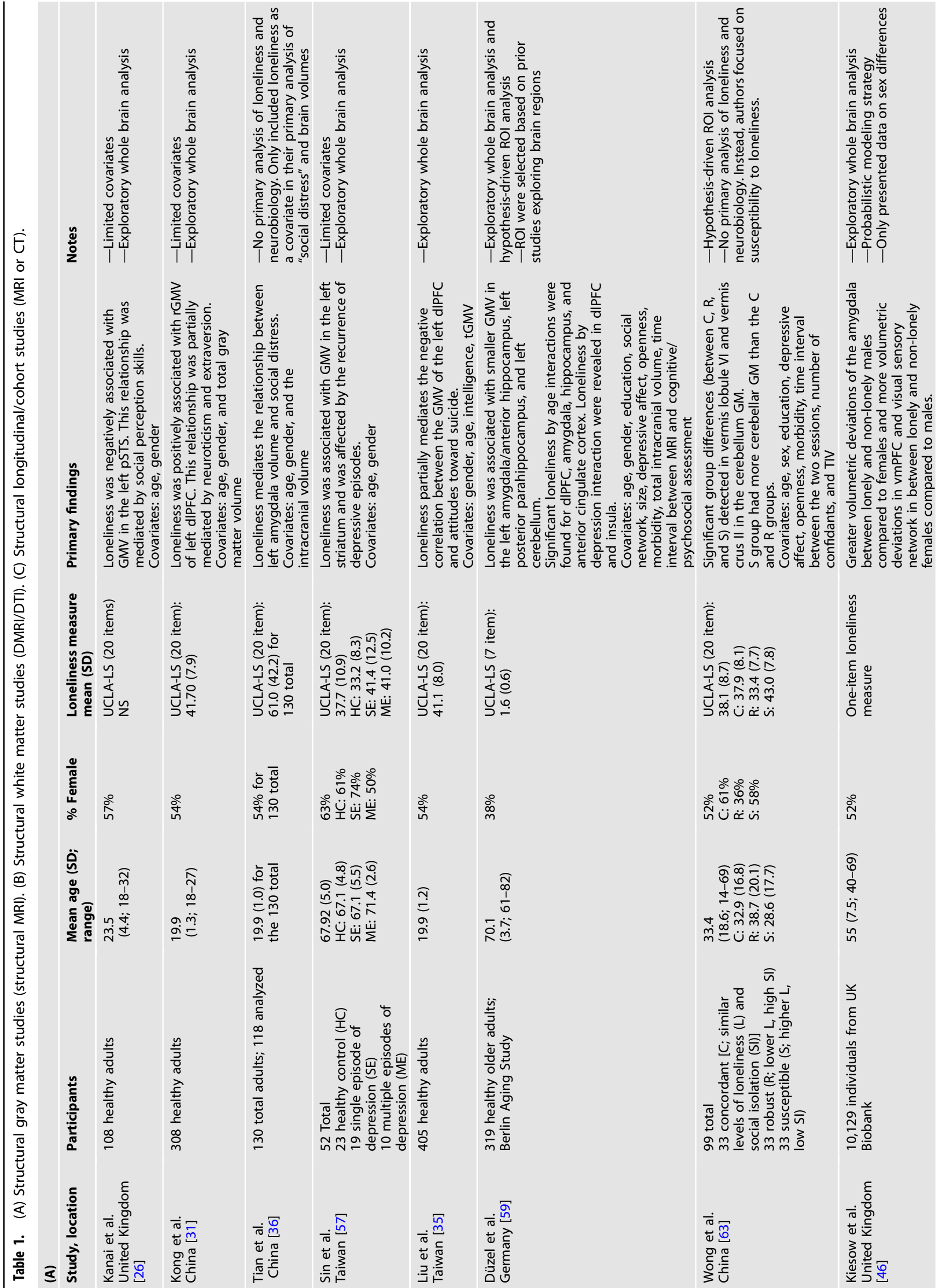




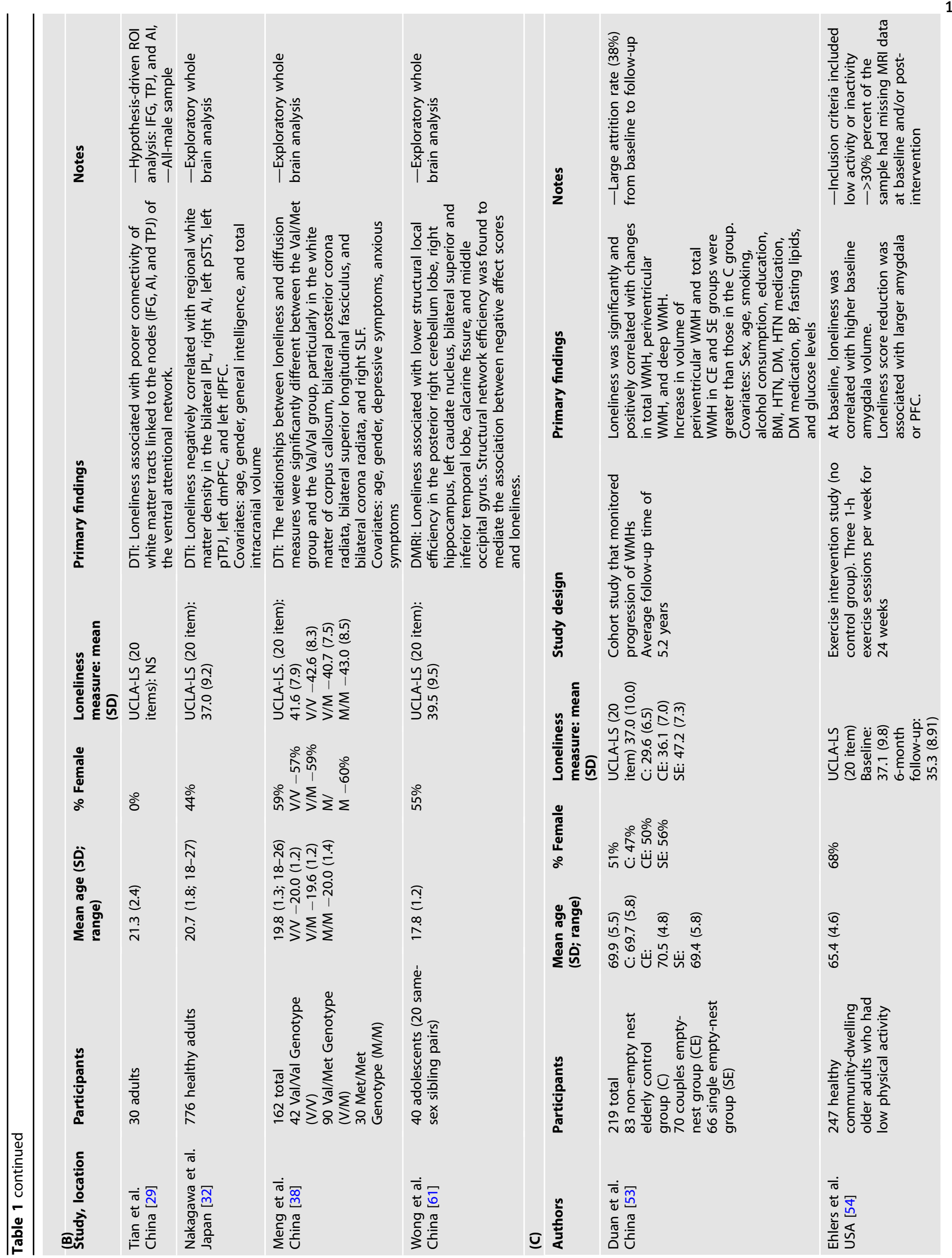




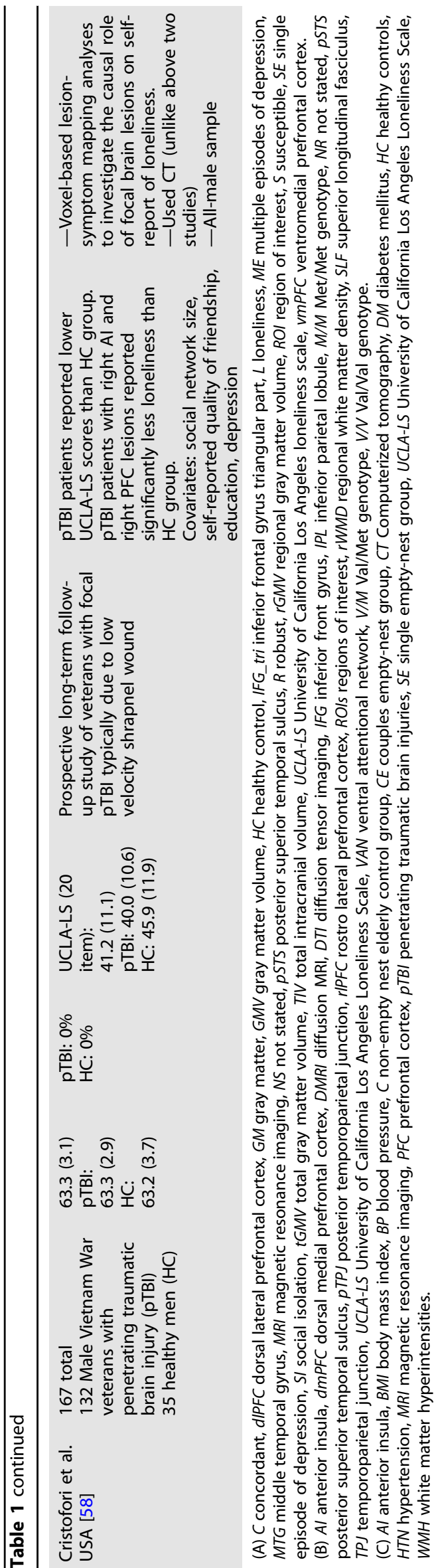

other networks, with decreased causal flow from affective to visual networks [39], and greater right visual cortex functional connectivity to posterior cerebellum when presented positive words in task-based fMRI [63]. Examining sex differences, the volume of visual sensory network (comprised of fusiform gyrus, posterior superior temporal sulcus, and middle temporal V5 area) deviated between lonely and non-lonely women but not men [46].

Attentional systems $(N=4)$. Investigations reported an association of loneliness with poorer connectivity of white matter tracts between the nodes of ventral attentional network [29] as well as differential activation of TPJ, a node in ventral attentional network. Loneliness was also linked to a weaker relationship between dorsal and ventral attentional networks indicating decreased ability to filter less relevant stimuli [39], as well as increased functional connectivity in brain regions associated with cinguloopercular network [37].

Default mode network (DMN) $(N=3)$. One study found that higher social dysfunction (defined by loneliness, higher social disability, and smaller social network) was associated with decreased DMN connectivity, specifically in anterior mPFC and posterior superior frontal gyrus [47], while another report showed that loneliness was associated with reduced DMN functional connectivity in older healthy individuals compared to those with late-life depression [51]. Using network analyses, one study found that among lonely individuals, overall resting-state network structures had increased integration (lower modularity) between attentional, visual, and default mode networks [43].

\section{Other studies}

Alzheimer's disease $(A D)$ pathology $(N=4)$. A prospective longitudinal study demonstrated that increases in loneliness were correlated with increases in white matter hyperintensities among non-demented older adults [53]. Two cross-sectional studies using PET imaging found a significant relationship between loneliness and higher amyloid burden, especially in APOE 4 carriers $[50,56]$, and greater tau pathology in right entorhinal cortex and right fusiform gyrus [56]). Another cohort study reported that the risk of the development of $A D$ was significantly higher in lonely (than non-lonely) individuals; however, global AD pathology ( $\beta$-amyloid plaques, neurofibrillary tangles, or cerebral infarction) in postmortem brains $(n=90)$ showed no significant relationship to loneliness [48].

EEG $(N=3)$. In two separate publications using a Stroop task, loneliness was associated with faster ERPs with negative social (compared to negative nonsocial) words and threatening social (compared to threatening nonsocial) images [30,33]. However, another report found no significant main effect of loneliness on error-related negativity (a component of ERP) when writing about a nostalgic event versus an ordinary experience [64].

Brain RNA expression $(N=2)$. Two studies of RNA expression in post-mortem brain tissue in nucleus accumbens and dIPFC $[52,55]$ identified hundreds of differentially expressed transcripts and genes among lonely compared to non-lonely individuals, especially genes associated with $\mathrm{AD}[52,55]$. The relationships between loneliness and white matter structures were significantly different between BDNF genotypes [38].

Other investigations $(N=3)$. One investigation reported loneliness was explained equally well by whole brain static and dynamic functional connectivity, in contrast to traits like cognitive functioning, which were explained better by dynamic connectivity [42]. Another study found loneliness was linked to altered brain activity in right inferior temporal gyrus on MRI and that the neural activity mediated the relationship between loneliness and 


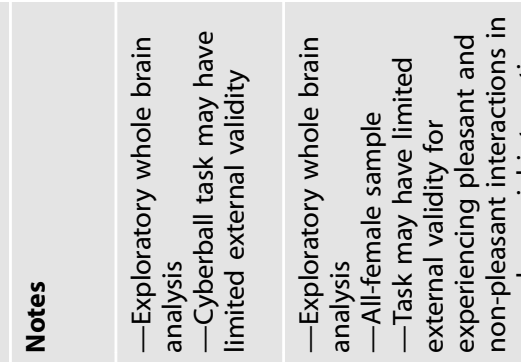

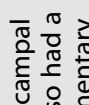

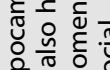

每

造

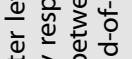

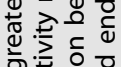

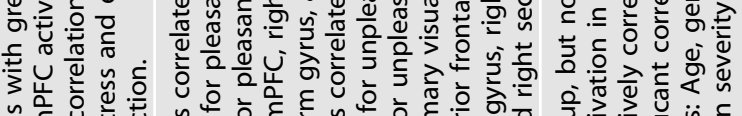

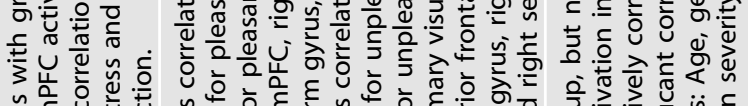

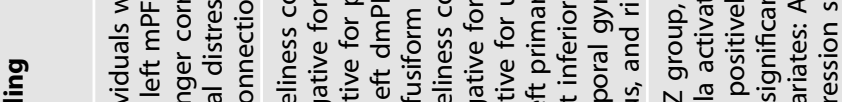

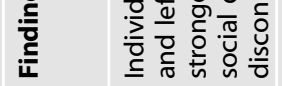

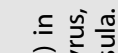

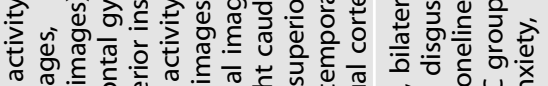

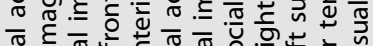

옥으는 는

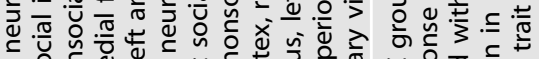

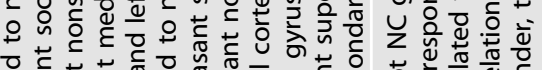

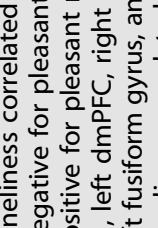

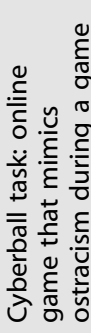

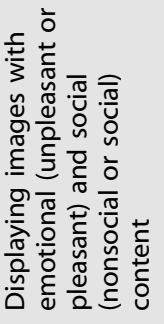

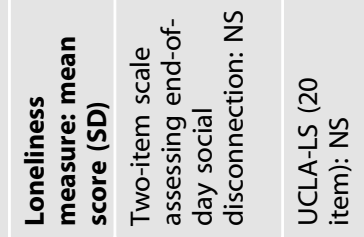

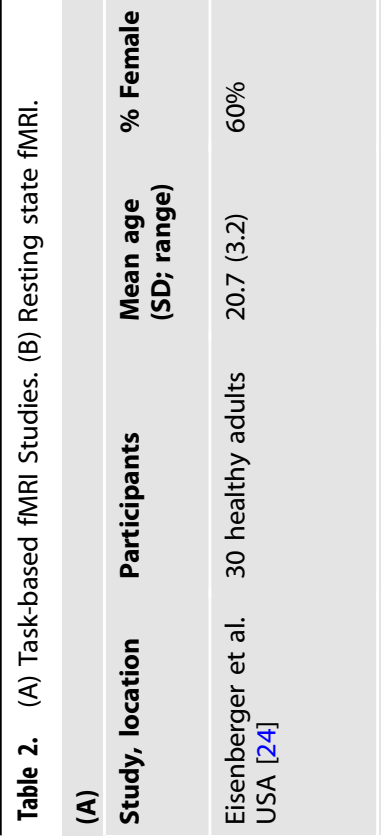

官

乏

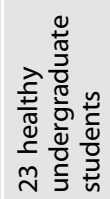

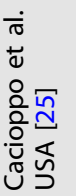

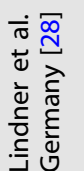

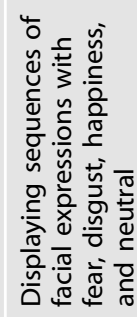

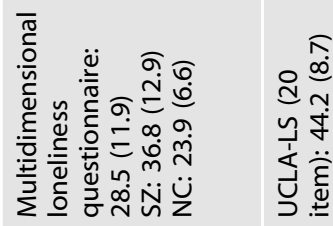

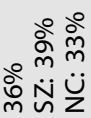

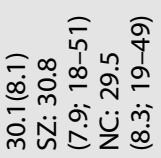

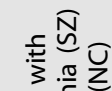

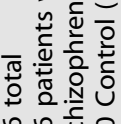

잉ํํ워

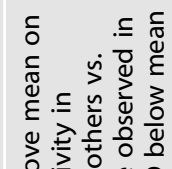

。ํำ

ผิ

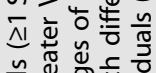

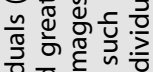

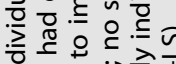

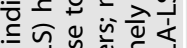

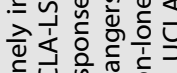

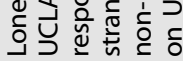

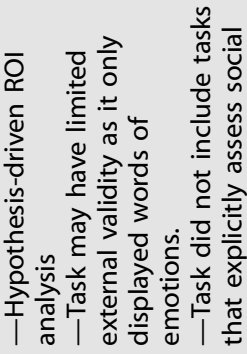

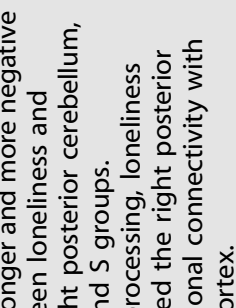

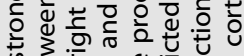
o.

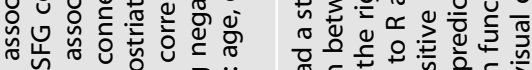

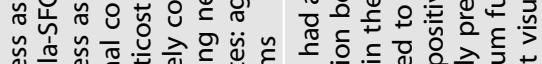

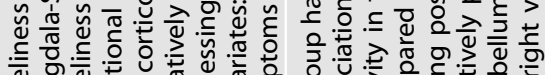

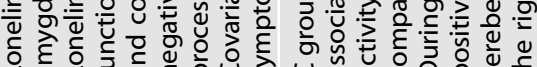

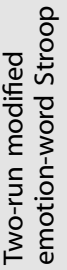

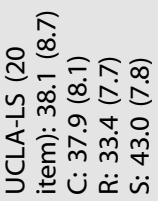

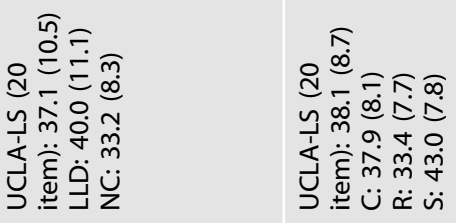

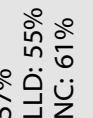

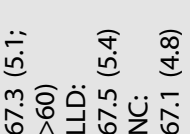

产蓄

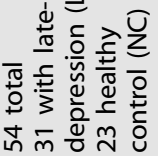

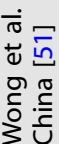

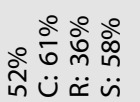

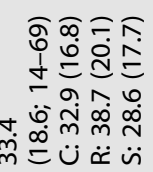

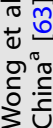

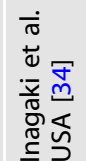




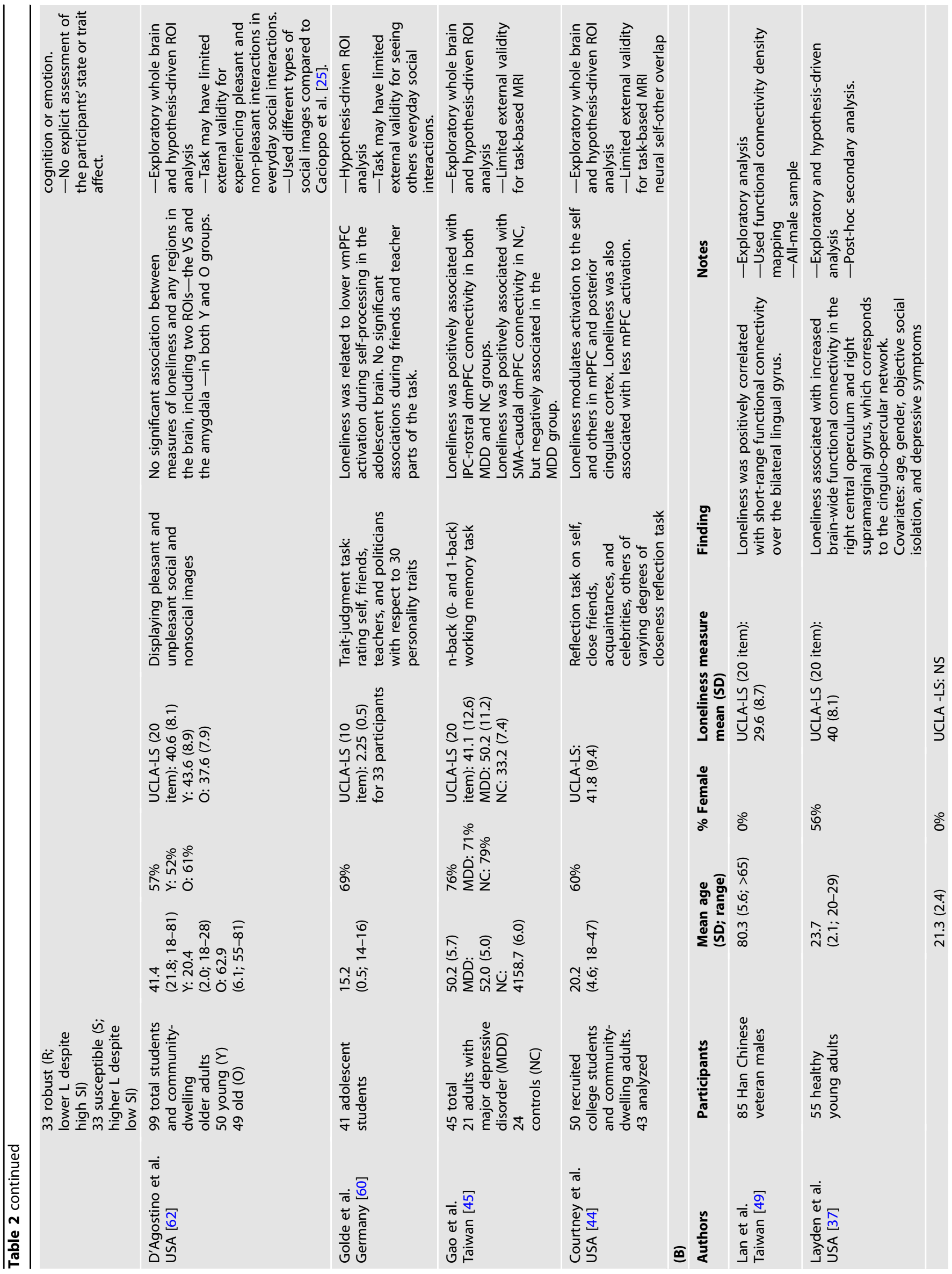




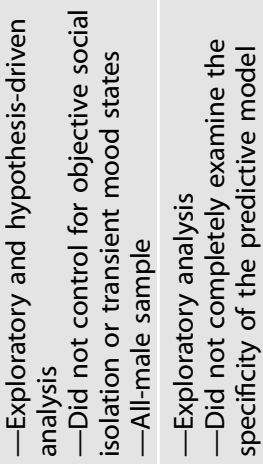

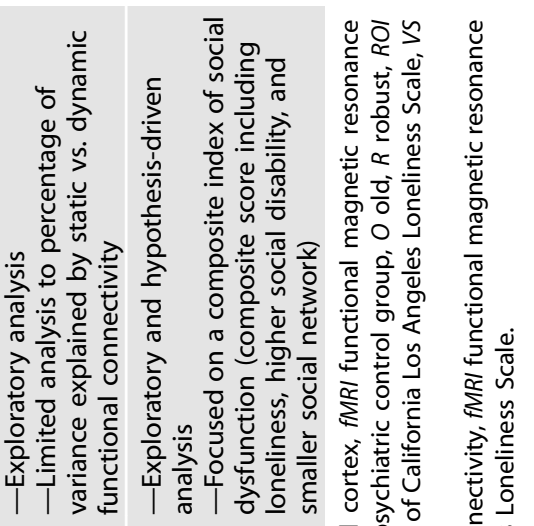

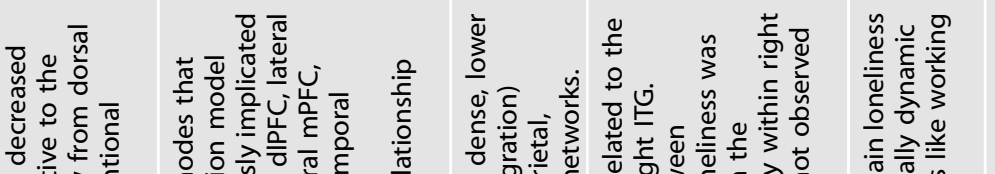

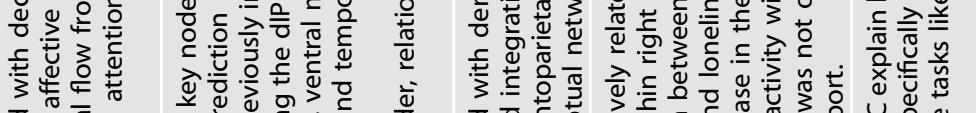

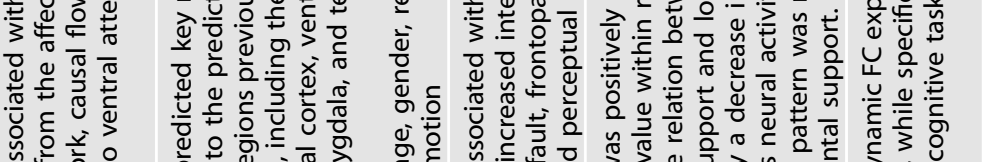

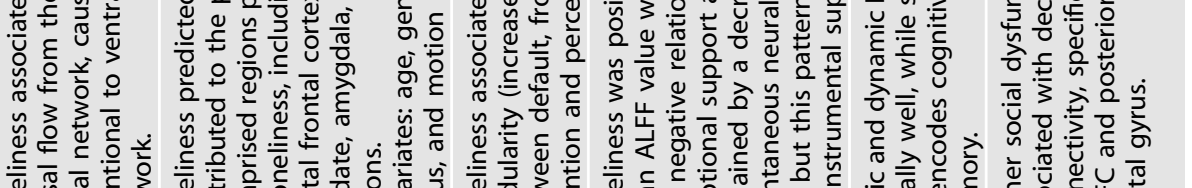

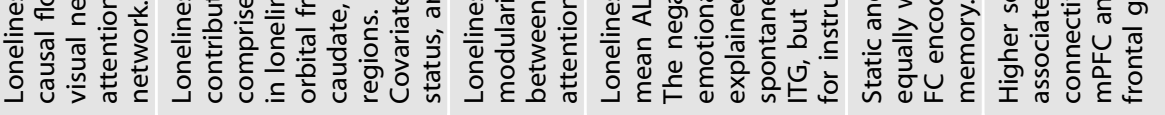
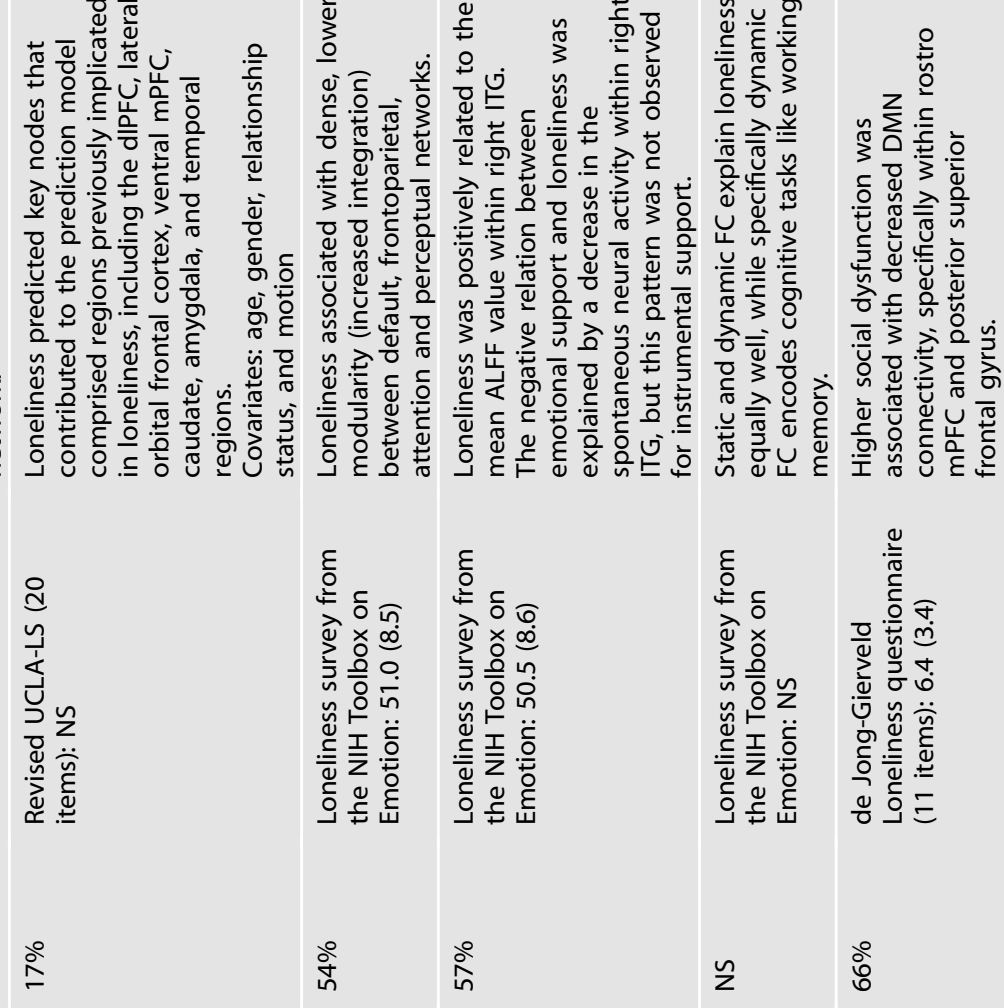

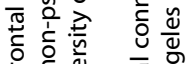

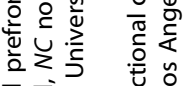

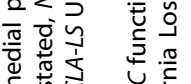

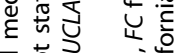

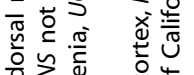

प्र

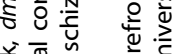

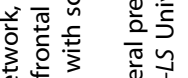

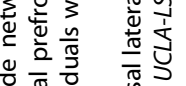

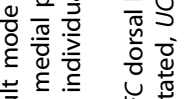

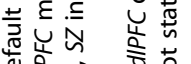

क्ष है छ

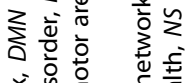

メ.

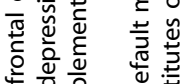

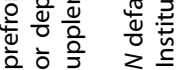

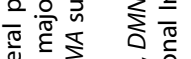

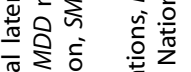

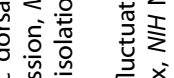

U人.

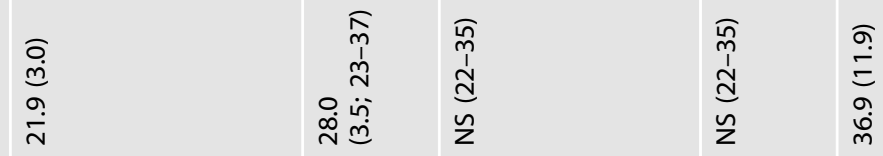

वे 웡

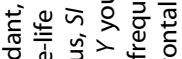

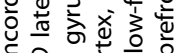

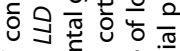

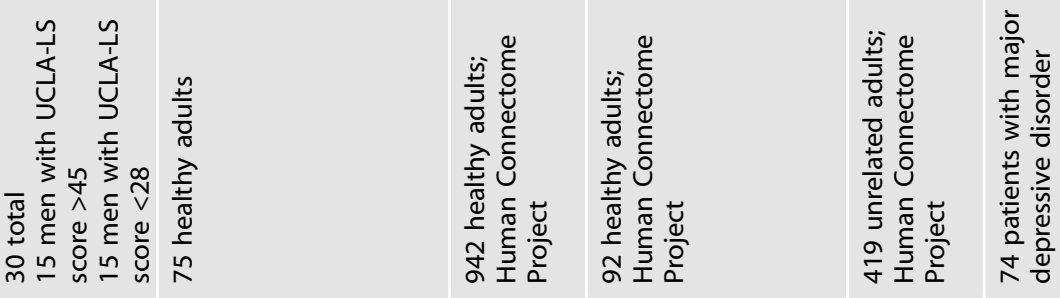

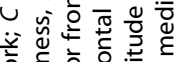

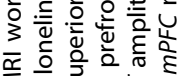

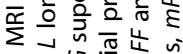

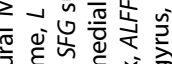

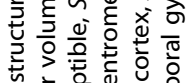

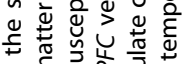

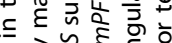

\begin{tabular}{|c|c|c|c|}
\hline 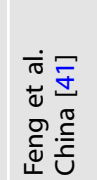 & 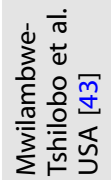 & 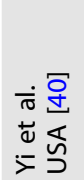 & 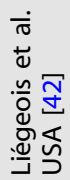 \\
\hline
\end{tabular}

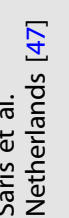

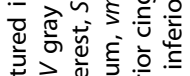

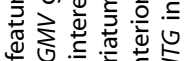

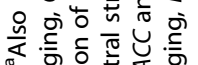

«. 
emotional support [40]. A longitudinal study reported that individuals with severe hearing impairment were lonelier and had a hypersensitive dopamine system in a SPECT scan pre- and post- amphetamine challenge, compared to people without hearing impairment [27].

\section{DISCUSSION}

To our knowledge, this is the first systemic review of neurobiology of loneliness. The 41 publications meeting our criteria show that, despite some mixed evidence, loneliness is associated with structural and functional differences in PFC, insula, hippocampus, amygdala, and posterior superior temporal cortex (Fig. 2, Supplementary Table 1), as well as attentional and visual networks and DMN. Drawing overall conclusions from this review is limited by the high heterogeneity of study methodologies and cohorts.

While there is no literature on loneliness in non-human animals, neurobiological correlates of social isolation have been examined in several animal studies and a few human studies. There are a few reviews focused on integrating animal social isolation and human research [11-13]. However, the social isolation literature in animal models focus more on changes in endocrinology, neurotransmitters, and oxidative stress, rather than neuroanatomical or functional brain differences [11]. These differing methodologies and paradigms make direct comparison challenging, though there are likely overlapping risk and protective factors for social isolation and loneliness.

The PFC mediates higher-order behaviors like emotional regulation and inhibitory control $[65,66]$. The dIPFC is implicated in working memory and executive function [67], and mPFC is implicated in self-referential processes such as self-criticism in social situations [68]. All 14 imaging studies examining PFC found associations of loneliness with structural (gray matter volume and white matter integrity) or functional components (activation with social vs. nonsocial images, and functional connectivity). These results are consistent with a previous review of animal studies of social isolation implicating PFC [13], and support loneliness as a complex socioemotional trait.

The insula somatic marker hypothesis states that insula receives and integrates information to create a "global emotional moment" [69]. Anterior insula plays a role in various behaviors including emotions, pain, and self-awareness [70]. In studies of gray matter, white matter connectivity, task-based activation, insula was reportedly associated with loneliness $[25,28,29,32,58,59]$. It has been proposed that social rejection activates similar regions as physical pain, as supported by bilateral anterior insula activation with feelings of loneliness $[70,71]$, although a more recent social rejection meta-analysis of $\mathrm{fMRI}$ studies did not find anterior insula involvement [72].

The amygdala is implicated in fear detection, positive stimuli processing, and emotional memories [73]. Four studies of amygdala reported some association between gray matter volume or task-based activation and loneliness, with possible age- and sex-interactions $[36,46,54,59]$. These findings are consistent with loneliness activating brain regions that support experiencing emotions. However, an fMRI study found no relationship with amygdala response to social stimuli [62].

The ventral striatum, which includes nucleus accumbens, plays a central role in reward reinforcement [74]. Three studies of ventral striatum response to social images produced divergent results $[25,34,62]$. A recently published fMRI study (published past our cutoff date) reported similar activation in substantia nigra/ ventral tegmental area (SN/VTA) among young adults undergoing either $10 \mathrm{~h}$ of social isolation or fasting from food [75], supporting loneliness as a state that motivates one to seek social interaction, much like hunger motivates one to seek food. Interestingly, lonely participants had less activation in the SN/VTA. Research based on social isolation in rodent models and social rejection in human experiments indicates that social isolation may alter social approach motivation [13], consistent with the findings that loneliness differentially alters ventral striatum and structures related to reward pathway.

The posterior superior temporal cortex, implicated in social cognition [76], was associated with loneliness in four studies $[25,26,32,61]$.

The hippocampus, known for its role in memory [77], and the cerebellum, known for sensorimotor coordination as well as cognitive and affective processes $[78,79]$ each had three papers that associated their function or structure with loneliness.

Attentional networks are responsible for effortful versus environmental, stimulus-driven control of attention, and are localized to distinct anatomical areas with specific cognitive functions [80]. Four publications reported that loneliness was associated with differences in ventral attentional (including TPJ), dorsal attentional, and cingulo-opercular networks, in terms of functional and effective connectivity [29, 37, 39, 43]. Attentional networks may be linked to hypervigilance and stress reactivity that are putatively involved in loneliness.

Visual systems are responsible for processing visual information. Five studies reported associations of loneliness with differences in primary and secondary visual cortex in terms of volume [46], functional connectivity [43], causal flow [39], or activation with social images $[25,63]$, supporting Cacioppo et al.'s hypervigilance theory of loneliness [6].

The DMN is active when the human brain is at rest and is implicated in mental representations of self across time and space, theory of mind, and pro-social behaviors [81]. Three studies showed an association between loneliness with DMN functional connectivity [43, 47, 51]. One report noted more dense, less modular connections between attentional, visual, and DM networks in lonely persons. A recent large $(n=\sim 40,000)$, multi-modal study (published after our cutoff date for inclusion in this review) reported increased volume and white matter structural connectivity as well as increased functional connectivity of the DMN in lonely individuals [82]. Together, these results suggest that higherorder brain regions localized to PFC and DMN may play a critical role in loneliness. The DMN may be differentially activated when we are thinking about others; however, dysregulated activity in DMN may contribute to rumination and negative feelings associated with loneliness.

Two EEG studies showed that lonely individuals had faster ERPs to negative or threatening stimuli $[30,33]$, consistent with the hypervigilance hypothesis of loneliness [6], while another report found no difference in ERPs with a nostalgia-related task [64].

Regarding AD markers, two PET studies reported greater amyloid and tau burden [50,56], and one MRI study reported progressive increase in white matter hyperintensities among lonely older adults [53]. However, one post-mortem study found no such differences in plaques, tangles, or infarcts [48]. Two studies extracting RNA from the brain identified differential ADrelated gene expression in lonely individuals $[52,55]$. The overall findings related to $A D$ align with meta-analytic evidence linking loneliness to increased risk of AD [83].

The brain regions highlighted in this review of loneliness may also have roles in other related constructs. For example, we have found a strong inverse correlation between loneliness and wisdom, especially its compassion component [15-17]. An overview of the neurobiology of wisdom has highlighted the major roles of PFC, especially dIPFC, vmPFC, anterior cingulate, and insula as well as amygdala $[21,84]$. One MRI study reported that loneliness and empathy were inversely associated with white matter density in lateral PFC, insula, and TPJ [32], while another MRI study found no links with gray matter density [26]. A recent EEG study demonstrated that loneliness and wisdom/compassion were related to contrasting modulations of cognitive processes, invoking similar (TPJ) and distinct (superior parietal vs. insula, 

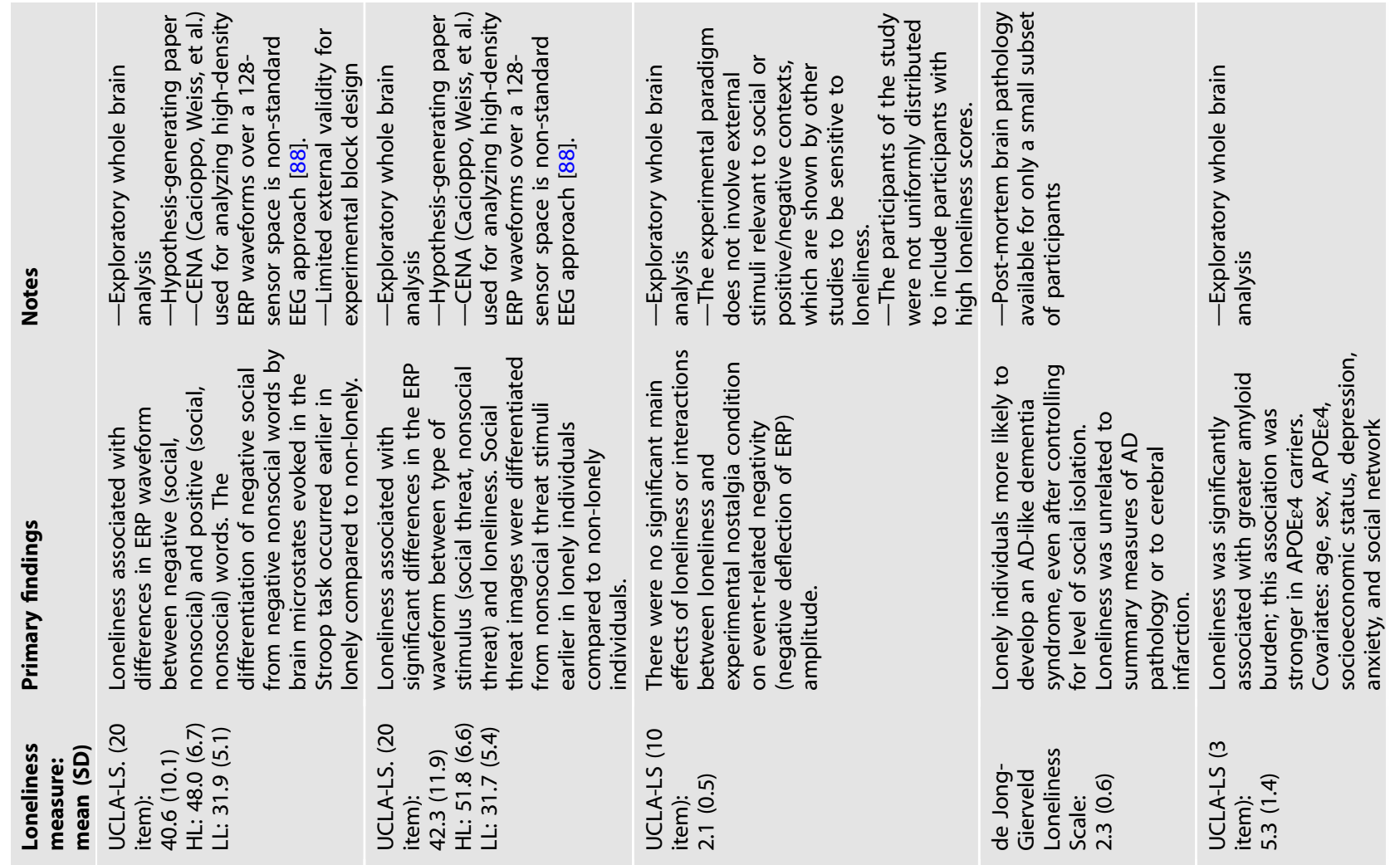

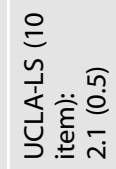
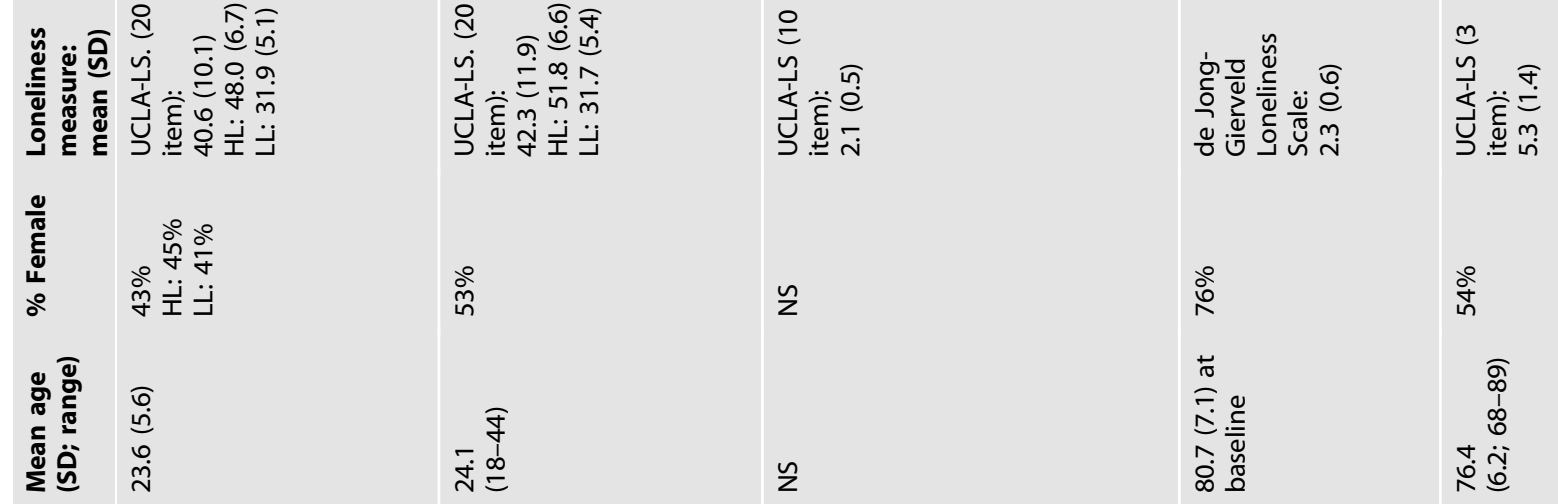

z

ڤ̊ํำ

率

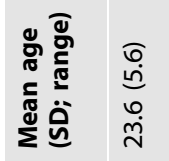

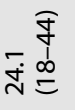

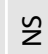

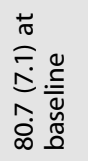

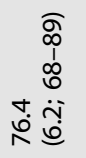
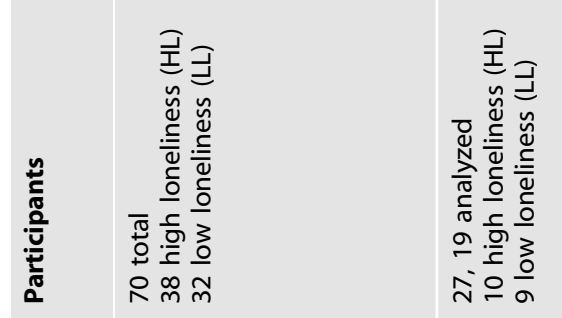

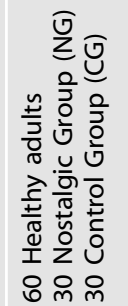

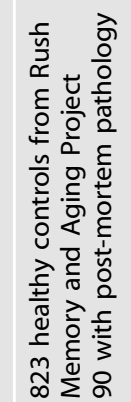

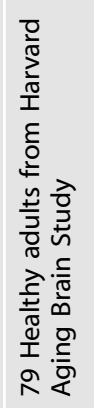

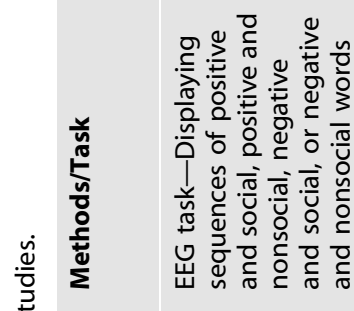
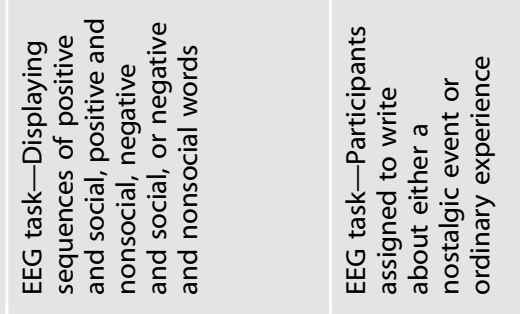

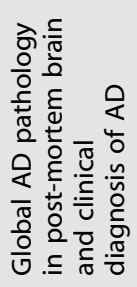
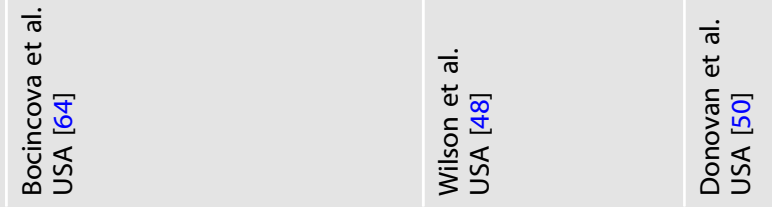


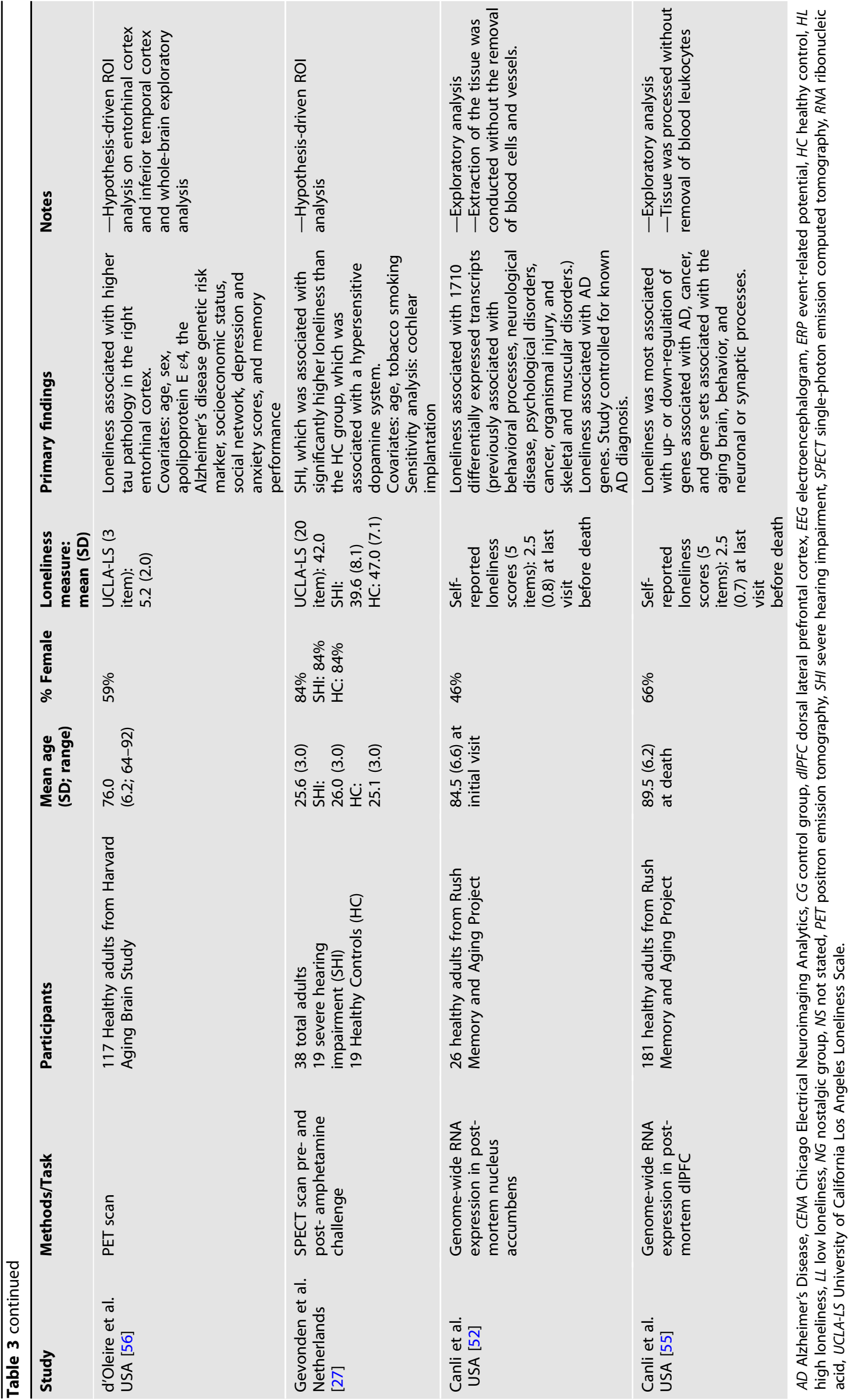



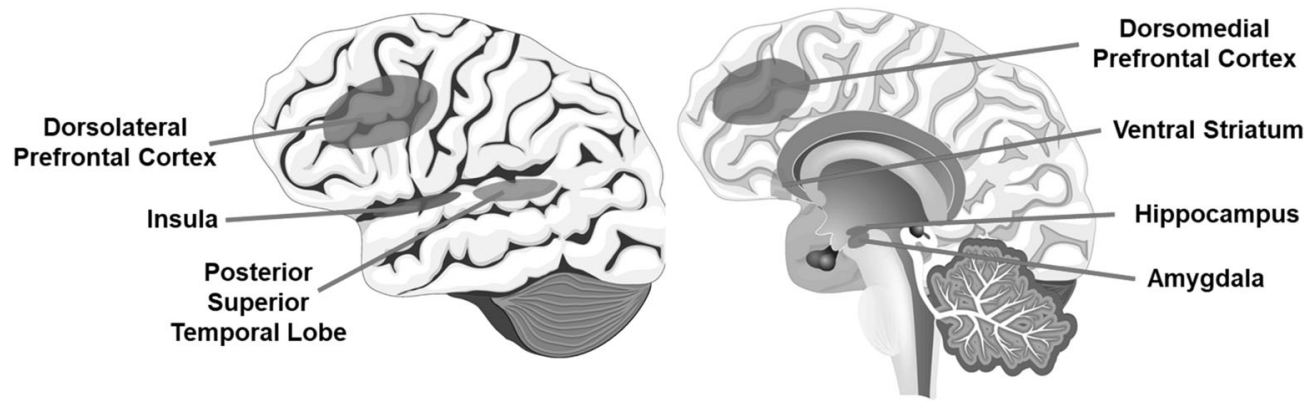

Fig. 2 Summary of the brain structures consistently implicated in loneliness. Left panel shows the lateral view of the brain with the relevant brain regions highlighted and labeled, while the right panel shows the sagittal view of the brain regions.

respectively) neural circuits in specific emotional contexts [85]. These relationships are correlational and warrant further study employing neurobiological perturbations.

\section{Limitations}

This review article as well as the included studies have limitations. It is possible that, despite our best efforts, we missed a few relevant papers. Also, we did not include articles in non-English languages, and $73 \%(30 / 41)$ of the reports came from the USA or China, thereby limiting the generalizability to other countries. Most investigations were cross-sectional, preventing causal inferences. There may be confounding factors that are driving these relationships. There is risk of gender bias in self-reported assessments of loneliness. While there are no agreed upon objective measures of loneliness, indirect partial objective measures may include sedentary behavior assessed with wearable activity trackers, life space using GPS data, and sleep disturbances using wearable sensors. The studies included are limited by varied methodologies and analysis techniques in the rapidly evolving field of social neuroscience. For example, EEG has remarkable temporal resolution, but poor spatial resolution while the reverse is true with fMRI [86]. Many studies were hypothesis-generating and used single neurobiological modalities. Though one study included over 10,000 participants from the UK Biobank registry study [46], the majority $(25 / 41 ; 61 \%)$ of the studies had fewer than 100 participants. Thus, most of the individual study findings are limited by small sample sizes, and overall generalizability may be low. Subject samples varied widely in sociodemographic characteristics, outcome measures, analysis protocols, and statistical methods, thereby precluding a metanalysis. It is not always clear if some brain regions not mentioned in the results had not been examined or were examined but not found to be significantly associated with loneliness.

Most studies only assessed and controlled for a small number of covariates such as demographic variables including age and sex. However, the complex psychosocial nature of loneliness extends beyond these basic demographic factors. Objective health status, environmental characteristics, stress, mental health, and personality traits are important confounders that were not included in many of the analyses. Only two studies had samples that could example the relationship of age (across the adult lifespan) with the loneliness-neurobiology associations $[62,63]$. Wong et al. reported reduced cerebellar gray matter with older age, while D'Agostino et al.reported no age-related findings. While Düzel et al. presented age-related findings, they were restricted to older adults 61-82 years [59]. Several, but not all, studies have examined depression as a confounder [38, 58, 59], and three case-control studies specifically examined the effect of loneliness for the neurobiological differences between people with depression and healthy controls $[45,51,57]$. However, despite their potential impact, other constructs including grief, prolonged grief disorder, mild cognitive impairment, substance use disorders, and various stress-related conditions were not assessed and analyzed in most studies of loneliness.

\section{Future directions}

This systemic review of neurobiology of loneliness identified how loneliness is linked to specific brain regions and networks, including PFC, insula, amygdala, hippocampus, attentional networks, and DMN, and a strong relationship with AD. However, researchers will need to replicate and expand the quantity and quality of these studies to understand the brain processes underlying loneliness. Moving forward, task-based neurocircuitry fMRI studies and multi-modal imaging studies have promise, due to the complexity of social cognition and functioning. These approaches would be well-suited to loneliness interventions to identify associated changes in connectivity. Future studies should also include large and diverse samples of well-characterized subjects followed longitudinally, with hypothesis-based approaches and appropriate multivariate statistical analyses, to examine the role of age and other relevant factors.

Studies should examine how the neurobiological findings are linked to other behaviors associated with loneliness-including sleep disturbances, sedentary behaviors, and limited life space. Assessments should include multi-modal assessments of social functioning-including use of social media, GPS-derived life space data, speech data, sleep, and ecological momentary assessments that examine loneliness as a state rather than a trait [87]. Neurobiological assessments that examine structural and functional integrity or harness neuromodulation techniques such as transcranial magnetic stimulation can also provide novel insights into brain alterations associated with loneliness. Furthermore, RCTs of novel loneliness interventions and associated neurobiological changes are warranted. Such research will pave the way for the development of therapeutic and preventive interventions to manage the behavioral pandemic of loneliness.

\section{REFERENCES}

1. Klinenberg E. "Is loneliness a health epidemic." New York Times. 9 Feb. 2018, https://www.nytimes.com/2018/02/09/opinion/sunday/loneliness-health.html? smid=em-share. Accessed 20 Mar 2020.

2. National Academies of Sciences, Engineering, and Medicine. Social Isolation and Loneliness in Older Adults: Opportunities for the Health Care System. Washington, DC: The National Academies Press; 2020. https://doi.org/10.17226/25663.

3. Valtorta NK, Kanaan M, Gilbody S, Ronzi S, Hanratty B. Loneliness and social isolation as risk factors for coronary heart disease and stroke: systematic review and meta-analysis of longitudinal observational studies. Heart 2016;102:1009-16.

4. Kuiper JS, Zuidersma M, Voshaar RCO, Zuidema SU, van den Heuvel ER, Stolk RP, et al. Social relationships and risk of dementia: a systematic review and metaanalysis of longitudinal cohort studies. Ageing Res Rev. 2015;22:39-57.

5. Beutel ME, Klein EM, Brähler E, Reiner I, Jünger C, Michal M, et al. Loneliness in the general population: prevalence, determinants and relations to mental health. BMC Psychiatry. 2017;17:1-7.

6. Hawkley LC, Cacioppo JT. Loneliness matters: a theoretical and empirical review of consequences and mechanisms. Ann Behav Med. 2010;40:218-27. 
7. Holt-Lunstad J, Smith TB, Baker M, Harris T, Stephenson D. Loneliness and social isolation as risk factors for mortality: a meta-analytic review. Perspect Psychol Sci. 2015;10:227-37.

8. Rico-Uribe LA, Caballero FF, Martín-María N, Cabello M, Ayuso-Mateos JL, Miret M. Association of loneliness with all-cause mortality: a meta-analysis. PloS one. 2018;13:e0190033.

9. Steptoe A, Shankar A, Demakakos P, Wardle J. Social isolation, loneliness, and allcause mortality in older men and women. Proc Natl Acad Sci. 2013;110:5797-801.

10. Cacioppo JT, Cacioppo S, Boomsma DI. Evolutionary mechanisms for loneliness. Cogn Emot. 2014;28:3-21.

11. Mumtaz F, Khan Ml, Zubair M, Dehpour AR. Neurobiology and consequences of social isolation stress in animal model-A comprehensive review. Biomed Pharmacother. 2018;105:1205-22.

12. Tomova $L$, Tye $K$, Saxe R. The neuroscience of unmet social needs. Soc Neurosci. 2021;16:221-31.

13. Cacioppo S, Capitanio JP, Cacioppo JT. Toward a neurology of loneliness. Psychol Bull. 2014;140:1464.

14. Lee EE, Depp C, Palmer BW, Glorioso D, Daly R, Liu J, et al. High prevalence and adverse health effects of loneliness in community-dwelling adults across the lifespan: role of wisdom as a protective factor. Int Psychogeriatr. 2019;31:1447-62.

15. Jeste DV, Di Somma S, Lee EE, Nguyen TT, Scalcione M, Biaggi A, et al. Study of loneliness and wisdom in 482 middle-aged and oldest-old adults: a comparison between people in Cilento, Italy and San Diego, USA. Aging Ment Health. 2020;1-11.

16. Jeste DV, Thomas ML, Liu J, Daly RE, Tu XM, Treichler EB, et al. Is spirituality a component of wisdom? Study of 1,786 adults using expanded San Diego Wisdom Scale (Jeste-Thomas Wisdom Index). J Psychiatr Res. 2021;132:174-81.

17. Nguyen TT, Lee EE, Daly RE, Wu TC, Tang Y, Tu X, et al. Predictors of loneliness by age decade: study of psychological and environmental factors in 2,843 community-dwelling americans aged 20-69 years. J Clin Psychiatry. 2020;81.

18. Ardelt M, Jeste DV. Wisdom and hard Times: The ameliorating effect of wisdom on the negative association between adverse life events and well-being. J Gerontol. 2018;73:1374-83.

19. Zacher H, Staudinger U.M. Wisdom and well-being. In Diener E, Oishi S \& Tay L (Eds), Handbook of well-being. Salt Lake City, UT: DEF Publishers; 2018. https:// nobascholar.com.

20. Judge TA, Ilies R, Dimotakis N. Are health and happiness the product of wisdom? The relationship of general mental ability to educational and occupational attainment, health, and well-being. J Appl Psychol. 2010;95:454-68.

21. Meeks TW, Jeste DV. Neurobiology of wisdom: a literature overview. Arch Gen Psychiatry. 2009;66:355-65.

22. Russell D, Peplau LA, Ferguson ML. Developing a measure of loneliness. J Pres Assess. 1978:42:290-94.

23. Hughes ME, Waite LJ, Hawkley LC, Cacioppo JT. A short scale for measuring loneliness in large surveys: results from two population-based studies. Res Aging. 2004;26:655-72.

24. Eisenberger NI, Gable SL, Lieberman MD. Functional magnetic resonance imaging responses relate to differences in real-world social experience. Emotion 2007;7:745-54.

25. Cacioppo JT, Hawkley LC. Perceived social isolation and cognition. Trends Cogn Sci. 2009;13:447-54.

26. Kanai R, Bahrami B, Duchaine B, Janik A, Banissy MJ, Rees G. Brain structure links loneliness to social perception. Curr Bio. 2012;22:1975-9.

27. Gevonden M, Booij J, van den Brink W, Heijtel D, van Os J, Selten JP. Increased release of dopamine in the striata of young adults with hearing impairment and its relevance for the social defeat hypothesis of schizophrenia. JAMA Psychiatry. 2014;71:1364-72.

28. Lindner C, Dannlowski U, Walhöfer K, Rödiger M, Maisch B, Bauer J, et al. Social alienation in schizophrenia patients: association with insula responsiveness to facial expressions of disgust. PLoS One. 2014;9:e85014.

29. Tian Y, Liang S, Yuan Z, Chen S, Xu P, Yao D. White matter structure in loneliness: preliminary findings from diffusion tensor imaging. Neuroreport 2014;25:843-47.

30. Cacioppo S, Balogh S, Cacioppo JT. Implicit attention to negative social, in contrast to nonsocial, words in the Stroop task differs between individuals high and low in loneliness: Evidence from event-related brain microstates. Cortex 2015;70:213-33.

31. Kong X, Wei D, Li W, Cun L, Xue S, Zhang Q, et al. Neuroticism and extraversion mediate the association between loneliness and the dorsolateral prefrontal cortex. Exp Brain Res. 2015;233:157-64.

32. Nakagawa S, Takeuchi H, Taki Y, Nouchi R, Sekiguchi A, Kotozaki Y, et al. White matter structures associated with loneliness in young adults. Sci Rep. 2015;5:17001.

33. Cacioppo S, Bangee M, Balogh S, Cardenas-Iniguez C, Qualter P, Cacioppo JT. Loneliness and implicit attention to social threat: a high-performance electrical neuroimaging study. Cogn Neurosci. 2016;7:138-59.
34. Inagaki TK, Muscatell KA, Moieni M, Dutcher JM, Jevtic I, Irwin MR, et al. Yearning for connection? Loneliness is associated with increased ventral striatum activity to close others. Soc Cogn Affect Neurosci. 2016;11:1096-101.

35. Liu H, Wang Y, Liu W, Wei D, Yang J, Du X, et al. Neuroanatomical correlates of attitudes toward suicide in a large healthy sample: a voxel-based morphometric analysis. Neuropsychologia 2016;80:185-93.

36. Tian X, Hou X, Wang K, Wei D, Qiu J. Neuroanatomical correlates of individual differences in social anxiety in a non-clinical population. Soc Neurosci. 2016;11:424-37.

37. Layden EA, Cacioppo JT, Cacioppo S, Cappa SF, Dodich A, Falini A, et al. Perceived social isolation is associated with altered functional connectivity in neural networks associated with tonic alertness and executive control. Neuroimage 2017;145:58-73.

38. Meng J, Hao L, Wei D, Sun J, Li Y, Qiu J. BDNF Val66Met polymorphism modulates the effect of loneliness on white matter microstructure in young adults. Biol Psychol. 2017;130:41-9.

39. Tian Y, Yang L, Chen S, Guo D, Ding Z, Tam KY, et al. Causal interactions in restingstate networks predict perceived loneliness. PloS one. 2017;12:e0177443.

40. Yi Y, Li LMW, Xiao Y, Ma J, Fan L, Dai Z. Brain activity mediates the relation between emotional but not instrumental support and trait loneliness. Soc Cogn Affect Neurosci. 2018;13:995-1002.

41. Feng $C$, Wang $L$, Li T, Xu P. Connectome-based individualized prediction of loneliness. Soc Cogn Affect Neurosci. 2019;14:353-65.

42. Liégeois R, Li J, Kong R, Orban C, Van De Ville D, Ge T, et al. Resting brain dynamics at different timescales capture distinct aspects of human behavior. Nat Commun. 2019:10:2317.

43. Mwilambwe-Tshilobo L, Ge T, Chong M, Ferguson MA, Misic B, Burrow AL, et al. Loneliness and meaning in life are reflected in the intrinsic network architecture of the brain. Soc Cogn Affect Neurosci. 2019;14:423-33.

44. Courtney AL, Meyer ML. Self-other representation in the social brain reflects social connection. J Neurosci. 2020:40:5616-27.

45. Gao M, Shao R, Huang CM, Liu HL, Chen YL, Lee SH, et al. The relationship between loneliness and working-memory-related frontoparietal network connectivity in people with major depressive disorder. Behav Brain Res. 2020:393:112776.

46. Kiesow H, Dunbar R, Kable J, Kalenscher T, Vogeley K, Schilbach L, et al. 10,000 social brains: sex differentiation in human brain anatomy. Sci Adv. 2020;6: eaaz1170.

47. Saris IMJ, Penninx B, Dinga R, van Tol MJ, Veltman DJ, van der Wee NJA, et al. Default mode network connectivity and social dysfunction in major depressive disorder. Sci Rep. 2020;10:194.

48. Wilson RS, Krueger KR, Arnold SE, Schneider JA, Kelly JF, Barnes LL, et al. Loneliness and risk of Alzheimer disease. Arch Gen Psychiatry. 2007;64:234-40.

49. Lan CC, Tsai SJ, Huang CC, Wang YH, Chen TR, Yeh HL, et al. Functional connectivity density mapping of depressive symptoms and loneliness in nondemented elderly male. Front Aging Neurosci. 2015;7:251.

50. Donovan NJ, Okereke OI, Vannini P, Amariglio RE, Rentz DM, Marshall GA, et al. Association of higher cortical amyloid burden with loneliness in cognitively normal older adults. JAMA Psychiatry. 2016;73:1230-37.

51. Wong NM, Liu HL, Lin C, Huang CM, Wai YY, Lee SH, et al. Loneliness in late-life depression: structural and functional connectivity during affective processing. Psychol Med. 2016;46:2485-99.

52. Canli T, Wen R, Wang X, Mikhailik A, Yu L, Fleischman D, et al. Differential transcriptome expression in human nucleus accumbens as a function of loneliness. Mol Psychiatry. 2017:22:1069-78.

53. Duan D, Dong Y, Zhang H, Zhao Y, Diao Y, Cui Y, et al. Empty-nest-related psychological distress is associated with progression of brain white matter lesions and cognitive impairment in the elderly. Sci Rep. 2017;7:43816.

54. Ehlers DK, Daugherty AM, Burzynska AZ, Fanning J, Awick EA, Chaddock-Heyman $L$, et al. Regional brain volumes moderate, but do not mediate, the effects of group-based exercise training on reductions in loneliness in older adults. Front Aging Neurosci. 2017;9:110.

55. Canli T, Yu L, Yu X, Zhao H, Fleischman D, Wilson RS, et al. Loneliness 5 years antemortem is associated with disease-related differential gene expression in postmortem dorsolateral prefrontal cortex. Transl Psychiatry. 2018;8:2.

56. d'Oleire Uquillas $F$, Jacobs HIL, Biddle KD, Properzi M, Hanseeuw B, Schultz AP, et al. Regional tau pathology and loneliness in cognitively normal older adults. Transl Psychiatry. 2018;8:282.

57. Sin ELL, Liu HL, Lee SH, Huang CM, Wai YY, Chen YL, et al. The relationships between brain structural changes and perceived loneliness in older adults suffering from late-life depression. Int J Geriatr Psychiatry. 2018;33:606-12.

58. Cristofori I, Pal S, Zhong W, Gordon B, Krueger F, Grafman J. The lonely brain: evidence from studying patients with penetrating brain injury. Soc Neurosci. 2019;14:663-75. 
59. Düzel S, Drewelies J, Gerstorf D, Demuth I, Steinhagen-Thiessen E, Lindenberger $\mathrm{U}$, et al. Structural brain correlates of loneliness among older adults. Sci Rep. 2019;9:13569.

60. Golde S, Romund L, Lorenz RC, Pelz P, Gleich T, Beck A, et al. Loneliness and adolescents' neural processing of Sself, friends, and teachers: Consequences for the school self-concept. J Res Adolesc. 2019;29:938-52.

61. Wong NML, Shao R, Yeung PPS, Khong PL, Hui ES, Schooling CM, et al. Negative affect shared with siblings is associated with structural brain network efficiency and loneliness in adolescents. Neuroscience 2019;421:39-47.

62. D'Agostino AE, Kattan D, Canli T. An fMRI study of loneliness in younger and older adults. Soc Neurosci. 2019;14:136-48.

63. Wong NML, Shao R, Wu J, Tao J, Chen L, Lee TMC. Cerebellar neural markers of susceptibility to social isolation and positive affective processing. Brain Struc Funct. 2019;224:3339-51.

64. Bocincova A, Nelson T, Johnson J, Routledge C. Experimentally induced nostalgia reduces the amplitude of the event-related negativity. Soc Neurosci. 2019;14:631-4.

65. Miller EK, Cohen JD. An integrative theory of prefrontal cortex function. Annu Rev Neurosci. 2001;24:167-202.

66. O'Reilly RC. The what and how of prefrontal cortical organization. Trends Neurosci. 2010;33:355-61.

67. Barbey AK, Koenigs M, Grafman J. Dorsolateral prefrontal contributions to human working memory. Cortex 2013;49:1195-205.

68. D'Argembeau A, Ruby P, Collette F, Degueldre C, Balteau E, Luxen A, et al. Distinct regions of the medial prefrontal cortex are associated with self-referential processing and perspective taking. J Cogn Neurosci. 2007;19:935-44.

69. Craig AD, Craig A. How do you feel-now? The anterior insula and human awareness. Nat Rev Neurosci. 2009;10:59-70.

70. Eisenberger NI. The pain of social disconnection: examining the shared neural underpinnings of physical and social pain. Nat Rev Neurosci. 2012;13:421-34.

71. Cacioppo S, Frum C, Asp E, Weiss RM, Lewis JW, Cacioppo JT. A quantitative metaanalysis of functional imaging studies of social rejection. Sci Rep. 2013;3:1-3.

72. Vijayakumar N, Cheng TW, Pfeifer JH. Neural correlates of social exclusion across ages: a coordinate-based meta-analysis of functional MRI studies. Neurolmage 2017;153:359-68.

73. LeDoux J. The amygdala. Curr Biol. 2007;17:R868-R74.

74. Lucas-Neto L, Neto D, Oliveira E, Martins H, Mourato B, Correia F, et al. Three dimensional anatomy of the human nucleus accumbens. Acta Neurochir. 2013;155:2389-98.

75. Tomova L, Wang KL, Thompson T, Matthews GA, Takahashi A, Tye KM, et al. Acute social isolation evokes midbrain craving responses similar to hunger. Nat Neurosci. 2020;23:1597-605.

76. Bigler ED, Mortensen S, Neeley ES, Ozonoff S, Krasny L, Johnson M, et al. Superior temporal gyrus, language function, and autism. Dev Neuropsychol. 2007;31:217-38

77. Burgess N, Maguire EA, O'Keefe J. The human hippocampus and spatial and episodic memory. Neuron 2002;35:625-41.

78. Grodd W, Hülsmann E, Lotze M, Wildgruber D, Erb M. Sensorimotor mapping of the human cerebellum: fMRI evidence of somatotopic organization. Hum Brain Mapp. 2001;13:55-73.

79. De Smet HJ, Paquier P, Verhoeven J, Mariën P. The cerebellum: its role in language and related cognitive and affective functions. Brain Lang. 2013;127:334-42.

80. Petersen SE, Posner MI. The attention system of the human brain: 20 years after. Annu Rev Neurosci. 2012;35:73-89.

81. Andrews-Hanna JR, Smallwood J, Spreng RN. The default network and selfgenerated thought: component processes, dynamic control, and clinical relevance. Ann N. Y Acad Sci. 2014;1316:29.

82. Spreng RN, Dimas E, Mwilambwe-Tshilobo L, Dagher A, Koellinger P, Nave G, et al. The default network of the human brain is associated with perceived social isolation. Nat Commun. 2020;11:6393.
83. Lara E, Martín-María N, De la Torre-Luque A, Koyanagi A, Vancampfort D, Izquierdo $A$, et al. Does loneliness contribute to mild cognitive impairment and dementia? A systematic review and meta-analysis of longitudinal studies. Ageing Res Rev. 2019;52:7-16.

84. Lee EE, Jeste DV. Neurobiology of wisdom. In: Sternberg RJ \& Glück J, editors. The Cambridge handbook of wisdom. Cambridge: Cambridge University Press; 2019. p. 69-93.

85. Grennan G, Balasubramani PP, Alim F, Zafar-Khan M, Lee EE, Jeste DV, et al Cognitive and Neural Correlates of Loneliness and Wisdom during Emotional Bias. Cereb Cortex. 2021;31:3311-22.

86. Harmon-Jones $\mathrm{E}$, Beer J. Introduction to social and personality neuroscience methods. in: Harmon-Jones E, Beer JS, Schultheiss OC, Johnstone T, Stanton SJ, editors. Methods in Social Neuroscience. Guilford Press: New York, NY; 2009. p. 1-9.

87. Shiffman S, Stone AA, Hufford MR. Ecological momentary assessment. Annu Rev Clin Psychol. 2008;4:1-32.

88. Cacioppo S, Weiss RM, Runesha HB, Cacioppo JT. Dynamic spatiotemporal brain analyses using high performance electrical neuroimaging: theoretical framework and validation. J Neurosci Methods. 2014;238:11-34.

\section{ACKNOWLEDGEMENTS}

The authors acknowledge the help of Dr. Dilip Jeste in providing administrative support for the research effort as well as Ms. Paula Smith in preparing and submitting the manuscript.

\section{AUTHOR CONTRIBUTIONS}

EEL and JAL contributed to the conception and design of the study. JAL wrote the first draft of the manuscript. JAL, ERM, KEY, MR were involved in the systematic review and data extraction. TTN, JM, BM, MT were involved in the data interpretation. JAL and EEL wrote sections of the manuscript and were involved in data interpretation. All authors contributed to manuscript revision, read, and approved the submitted version. JAL and EEL had full access to all the data in the study and take responsibility for the integrity of the data and the accuracy of the data analysis.

\section{FUNDING AND DISCLOSURES}

This study was supported, in part, by the National Institute of Mental Health [NIMH R01MH094151 (PI: Dilip V. Jeste), NIMH K23MH119375-01 (PI: EEL), NIMH K23 MH118435 (PI: TTN)], by the VA San Diego Healthcare System, by the Stein Institute for Research on Aging (Director: Dilip V. Jeste, MD) at the University of California San Diego, and by IBM Research Al through the Al Horizons Network. The authors have no conflicts of interest with the work described.

\section{ADDITIONAL INFORMATION}

Supplementary information The online version contains supplementary material available at https://doi.org/10.1038/s41386-021-01058-7.

Correspondence and requests for materials should be addressed to E.E.L.

Reprints and permission information is available at http://www.nature.com/ reprints

Publisher's note Springer Nature remains neutral with regard to jurisdictional claims in published maps and institutional affiliations. 